Uluslararası Mühendislik

\title{
Design, Fabrication, and Characterization of a Water-Cooled Electromagnet and Magnetic Field Mapping
}

\author{
Haci Sogukpinar ${ }^{\text {iD }}$, Bayram Tali ${ }^{2}$ iD, Mahmut Tepe $^{3}$ iD \\ ${ }^{1} 1$ Department of Electric and Energy, Vocational School, University of Adiyaman, Adiyaman 02040, TURKEY \\ ${ }^{2}$ Department of Physics, Faculty of Art and Sciences, University of Adiyaman, Adiyaman, 02040, TURKEY \\ ${ }^{3}$ Graduate School of Sciences, University of Adiyaman, Adiyaman, 02040, TURKEY
}

Kabul / Accepted: 21/02/2020

Çevrimiçi Basım / Published Online: 18/03/2020

Son Versiyon/Final Version: 30/06/2020

\begin{abstract}
In this paper, a prototype solenoid magnet was designed and fabricated to be used in Micro Wave Discharge Ion Sources in the Turkish Accelerator Center Proton Accelerator Project and magnetic field mapping was performed with an experiment and also investigation was performed by using (FEM) finite element method. For this, a water-cooled electromagnet was designed and fabricated from 1010 steel with a thickness of $10 \mathrm{~cm}$. Magnetic field sensor was connected to a newly designed robotic arm and 2570 magnetic field intensity measurements were performed in the core of magnet with the size of $20 \mathrm{~cm}$ diameter and $10 \mathrm{~cm}$ depth of cylinder. The robotic arm was driven by three stepper motors and the measurement was automated with the Arduino physical programming platform. The UGN3503U Hall Effect sensor was used to measure the magnetic field and for the data processing ROOT was used. The magnetic field distribution inside the core of the solenoid magnet was investigated by using the finite element method. The commercial version of The Flexible Generalized Minimal Residual method (FGMRES) solver (built-in COMSOL) was used for the numerical part and the magnetic field was calculated and compared with the experiment to correlate simulation accuracy of this study.
\end{abstract}

\section{Key Words}

"solenoid magnet, finite element method, water-cooled magnet, robotic arm, hall sensors." 


\section{Introduction}

Today, more than 30.000 particle accelerators are operating around the world, and only $1 \%$ of them are high-energy investigation machines bigger than $1 \mathrm{GeV}$, about $44 \%$ for radiotherapy, $41 \%$ for ion implantation, $9 \%$ for industrial operation and investigation, and $4 \%$ for biomedical and other low-energy study applications (Feder, 2010). There are 12 cyclotrons in Turkey and all 150 low energy electron linear accelerator for radiation therapy used for the generation of medical isotopes in Turkey (TAEK, 2012). However, detailed studies on the research-based accelerator system have started in 1996 and a feasibility report was completed in 2001 (TAC, 2019). TAC (Turkish Accelerator Center) project consists of 5 subprojects and they are SC linac based IR FEL \& Bremsstrahlung facility in the span of 15-40 MeV, a third-generation light source based on a special $3 \mathrm{GeV}$ electron synchrotron, a fourth-generation light source 1 $\mathrm{GeV}$ electron LINAC, mass-energy center $3.77 \mathrm{GeV}$ and high electron-positron collider with power $(2 \mathrm{GeV})$ and high flux proton accelerator facility. Turkish Accelerator Center (TAC-PAF), both normal conductor is based on the $1 \mathrm{MW}$ and 2-GeV proton LINAC includes both superconducting accelerator structures (Algin et al., 2014). For the Proton Accelerator, Facility MDIS (Microwave Discharge Ion Source) ion source was planned to be used. 875 gauss is required to satisfy the ECR resonance condition in the ion source. Solenoid magnet with the iron jacket was designed and built for the confinement of the plasma produced by an electron cyclotron resonance source operating at $2.45 \mathrm{GHz}$ (Jain et al., 2010). Magnetic field optimization was performed with Poison software and magnetic field mapping was presented. The magnetic field was calculated with a computer program (INTMAG) and compared with analytical solutions and precision measurements (Becker, 1990) by integrating the contributions of real filaments and the results are found to be more accurate than from any finite difference element method program. A high-performance integrated solenoid inductor with a magnetic core was fabricated and investigated. An inductance above $70 \mathrm{nH}$ was achieved while keeping the coil resistance below $1 \mathrm{ohm}$ and the device area below $1 \mathrm{~mm}$ using a solenoid design with a single magnetic layer (Lee et al., 2008). Solenoid magnets generate a nearly smooth field profile that is less than 1000 gausses that exceed the ECR (electron cyclotron resonance) resonance area (875 gauss) along the entire plasma chamber axis length. Owing to the external iron components, the magnetic field drops rapidly in the extraction zone (Maimone et al., 2011).

In this study, a solenoid coil with an iron jacket was designed for MDIS ion source in Turkish Accelerator Center Proton Accelerator and domestic production possibilities and the formation of domestic knowledge were investigated and also magnetic field mapping was performed in the core of the magnet. For the numerical part, commercial software COMSOL was used to investigate magnetic field patterns inside the cylinder and obtained data were compared with the experiment to correlate simulation accuracy of this approach.

\section{Water-cooled Solenoid Magnet}

The MDIS ion source is planned to be used in the Turkish Accelerator Center Proton Accelerator Project. This ion source needs a solenoid magnet, capable of producing 0.05-0.1 T of the magnetic field. For this, the iron yoke was made from 1010 steel with $10 \mathrm{~mm}$ thickness. The inner and outer diameters of the steel body are $200 \mathrm{~mm}$ and $1000 \mathrm{~mm}$, respectively as it is shown in Fig. 1(a). For the winding, hollow, cylindrical copper tubes of $1 \mathrm{~mm}$ wall thickness were used. The outer diameter of the copper pipe is $6 \mathrm{~mm}$ and the inner diameter is $4 \mathrm{~mm}$. On the surface of the cylinder, 335 windings were made and the surface was covered with steel sheet. In order to prevent heating during operation, water is circulated through the pipes with the help of a water pump. The final fabrication image of the solenoid magnet with the water inlet and outlet, electrical connections and other components is given in Fig.1(b).

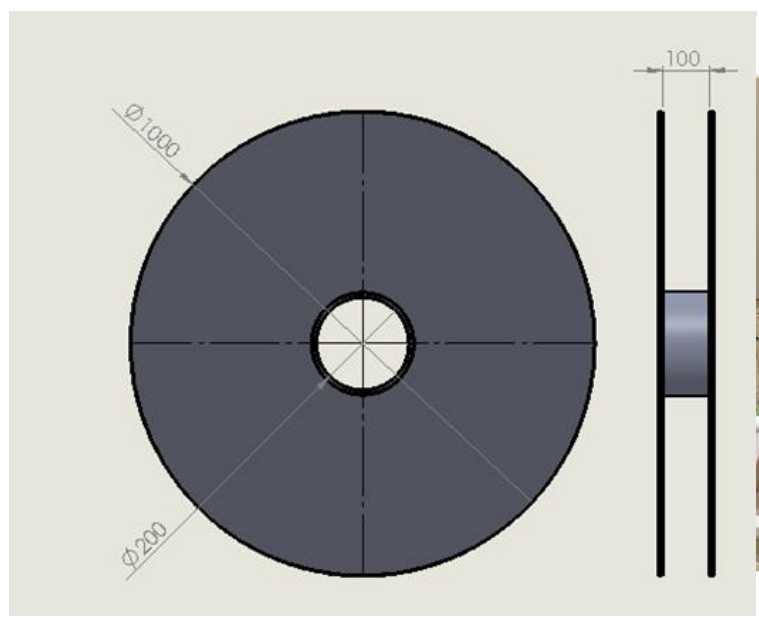

(a)

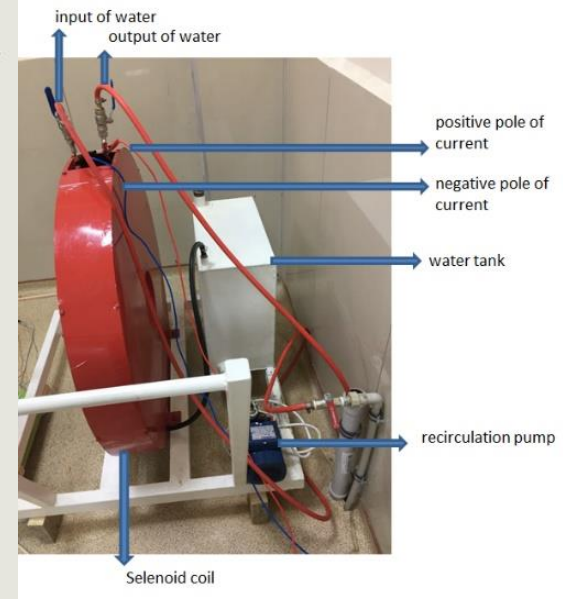

(b)

Figure 1. Design (a) and fabrication (b) of Solenoid magnet. 


\section{3D Robotic Arm}

The internal volume of the cylinder where the magnetic field was mapped is $4396 \mathrm{~cm}^{3}$. The robotic arm should be able to scan the entire volume in three dimensions. The movement of the robot arm is carried out with three-step motors. The stepper motors were connected to the arms that can move in three dimensions on the sled. In one step, stepper motors can move the arm $2 \mathrm{~mm}$ in the commanded direction (X-Y-Z). In order for the stepper motor to operate, the motor needs 9 volts and $0.1 \mathrm{~A}$. These stepper motors are controlled by the Arduino (UNO) microprocessor. UGN3503U Hall effect sensor was used to measure the magnetic field. This sensor can accurately detect small changes in the magnetic flux. In order to make magnetic field measurements more accurate, the software was commanded to hold the head for 10 seconds delay. Then, 10 measurements were taken at the intervals of one second then they are averaged and recorded as the measurement result for that coordinate point. The calibration set was made in preliminary measurement so that the sensor can reach the target coordinates without error. The $\mathrm{x}, \mathrm{y}$, and $\mathrm{x}$-axis were defined to the system as it is shown in Fig.2.

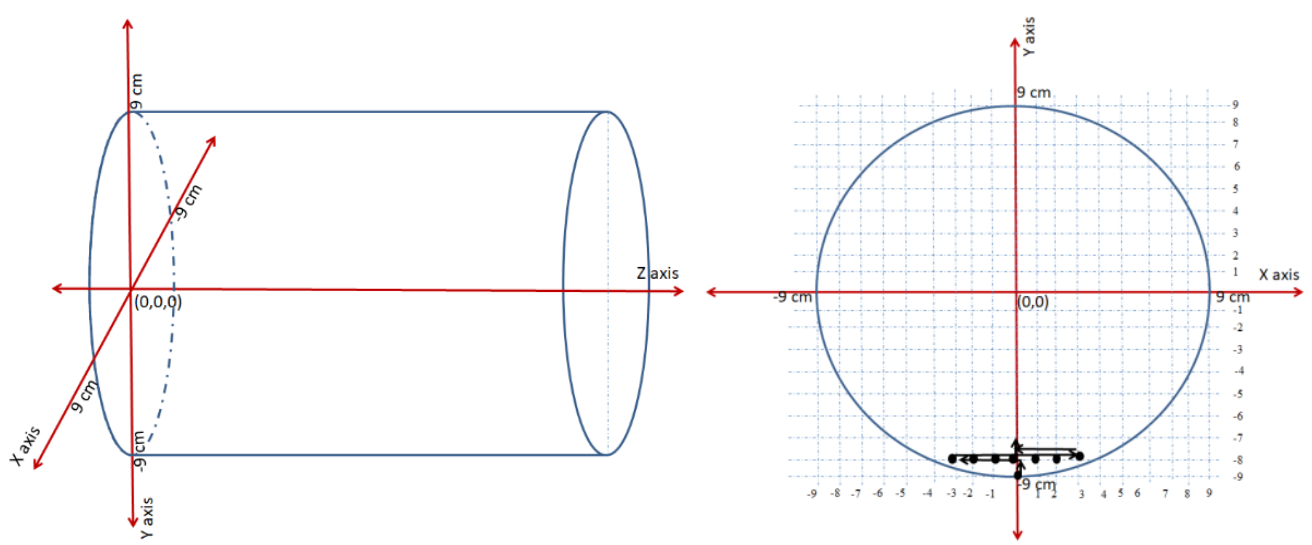

Figure 2. $x-y-z$ coordinate axis $(\mathrm{cm})$ of the cylinder; figure left to right: side (a) and front view (b).

Each coordinate has $10 \mathrm{~s}$ standby time for the sensor to reach saturation. Then, 10 measurements were taken at $1 \mathrm{~s}$ intervals and the results were recorded after averaging. For example, for the first layer measurements, the sensor was fixed behind the $\mathrm{z}=0$ coordinate $(\mathrm{z}=-2 \mathrm{~cm})$ and $\mathrm{y}=-9 \mathrm{~cm}$ and only one measurement was taken at $\mathrm{x}=0$ and there is no other point in this coordinate. Then head moved $1 \mathrm{~cm}$ in the $\mathrm{y}$-axis and comes to the point at $\mathrm{y}=-8 \mathrm{~cm}$ and measurement were taken through the $\mathrm{x}$-axis $(\mathrm{x}=-3,-2,-1,0,1,2,3)$. Then head moves to $y=-7$ and scans related $x$-axis. In this way, the $z$-axis was held constant and the $y$-axis was scanned from -9 to +9 , and the values for each $\mathrm{x}$-axis were measured and saved for each stage. Then the $\mathrm{z}$-axis was shifted by one unit $(2 \mathrm{~cm})$ and the measurements were continued and a totally of 2570 magnetic field data were measured and saved. Two measurements were taken in each determined $\mathrm{x}, \mathrm{y}, \mathrm{z}$ coordinate axis. The first one was performed without applying current to the solenoid magnet to measure the background magnetic field and the second one was performed with $8 \mathrm{~A}$ current.

\section{Experimental Results}

The magnetic field in the center of the magnet was mapped with the help of a Hall Effect sensor connected to a robotic arm. Two measurements were taken in each determined $\mathrm{x}, \mathrm{y}, \mathrm{z}$ coordinate axis point. The first one was performed without applying current to the solenoid magnet to measure the background magnetic field and the second one was performed with $8 \mathrm{~A}$ current. A modular scientific software toolkit, ROOT was used for data processing. ROOT is an open-access software. It provides all the functionalities needed to deal with big data processing, statistical analysis, visualization, and storage. When the root macro runs for all data, 3D magnetic field measurement data coordinates are given in Fig. 3. 


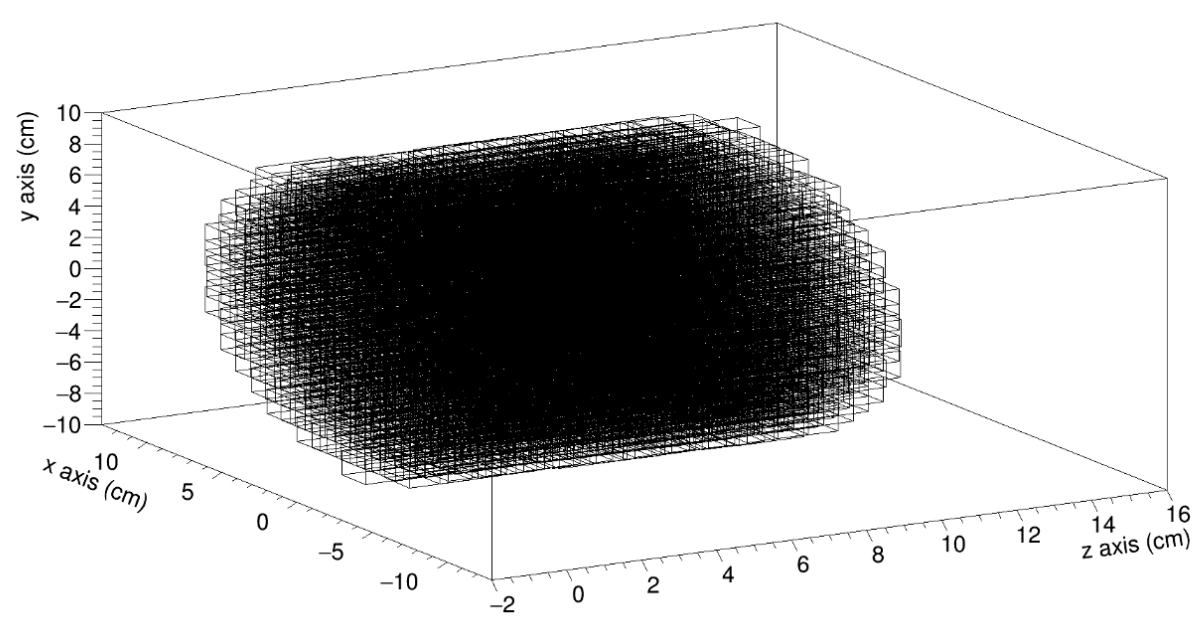

Figure 3. 3D magnetic field data

Magnetic field data were analyzed in three different ways. The first one was measured magnetic field data (B0) without applying an electrical current, the second one was total magnetic field data (B1) measured by applying current and the last one was the difference of the data set (B1-B0). Statistical presentation of magnetic field measurement data (B0, B1, B1-B0) for x, y and z-axis are as shown in Fig. 4.257 measurements were taken for the $\mathrm{z}=-2(\mathrm{x}, \mathrm{y},-2)$ coordinate according to Fig. 4 and the average magnetic field without applying current were measured as 3.7 Gauss but when the current is applied, the average magnetic field was measured as 8.09 Gauss. In order to measure the magnetic field only by the electric current, both values should be subtracted. The average magnetic field produced by the only electric current was calculated as 4.375 Gauss. The two-dimensional ( $y-x)$ appearance of the magnetic field data for the layer at $\mathrm{z}=0(\mathrm{x}, \mathrm{y}, 0)$ is shown in Fig. 5.
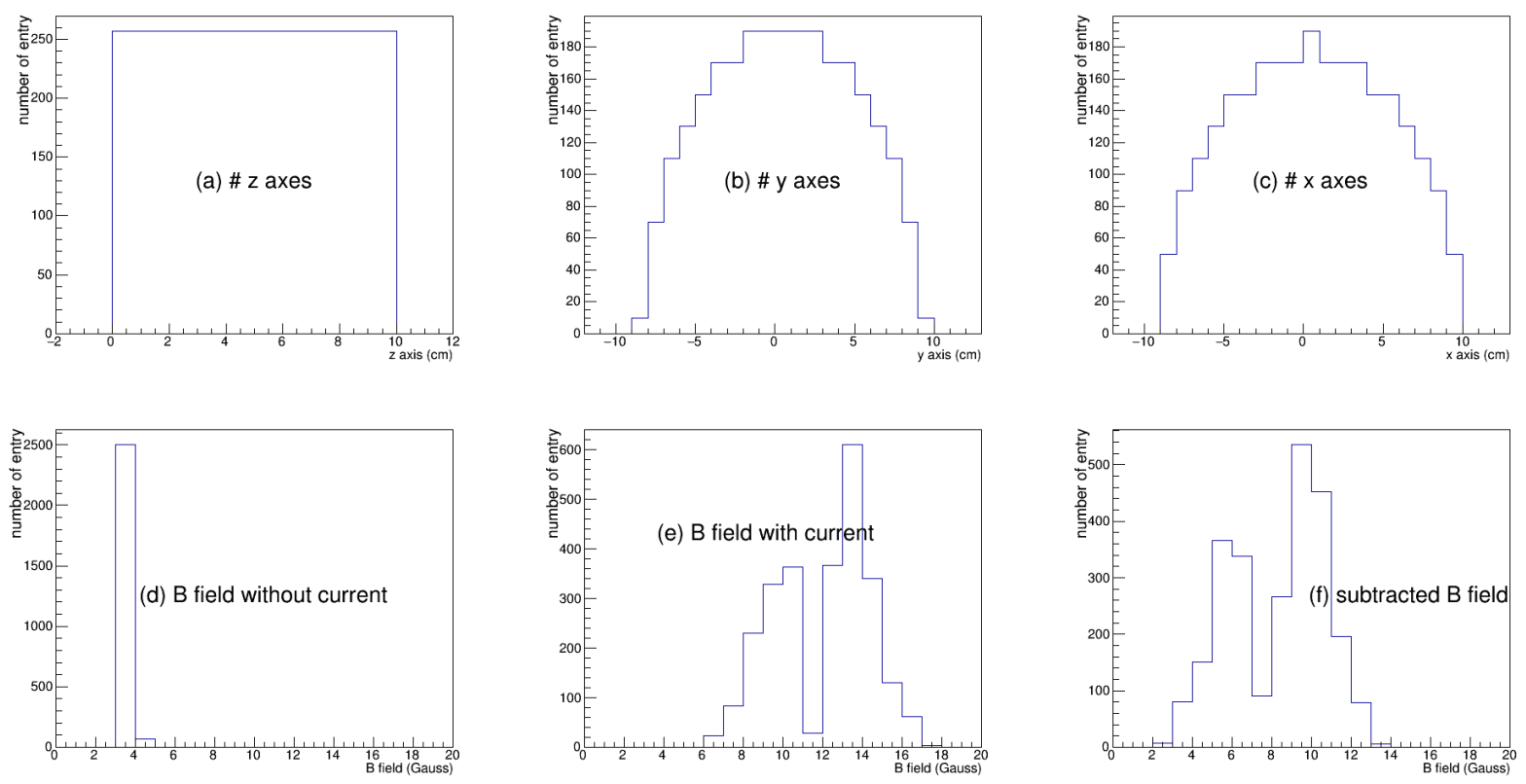

Figure 4. Statistical presentation of magnetic field measurement data for $\mathrm{x}, \mathrm{y}$, and $\mathrm{z}$-axis; figure a to $\mathrm{f}$ : total number of measurement point (a) with respect to $\mathrm{z}$-axis, total number of measured point in on $\mathrm{y}$-axis (b), total number of measured point in on $\mathrm{x}$-axis (c), number of point measured for background magnetic field (d), number of point measured with current (e), and number of point for the magnetic field subtracted from background magnetic field (f). 

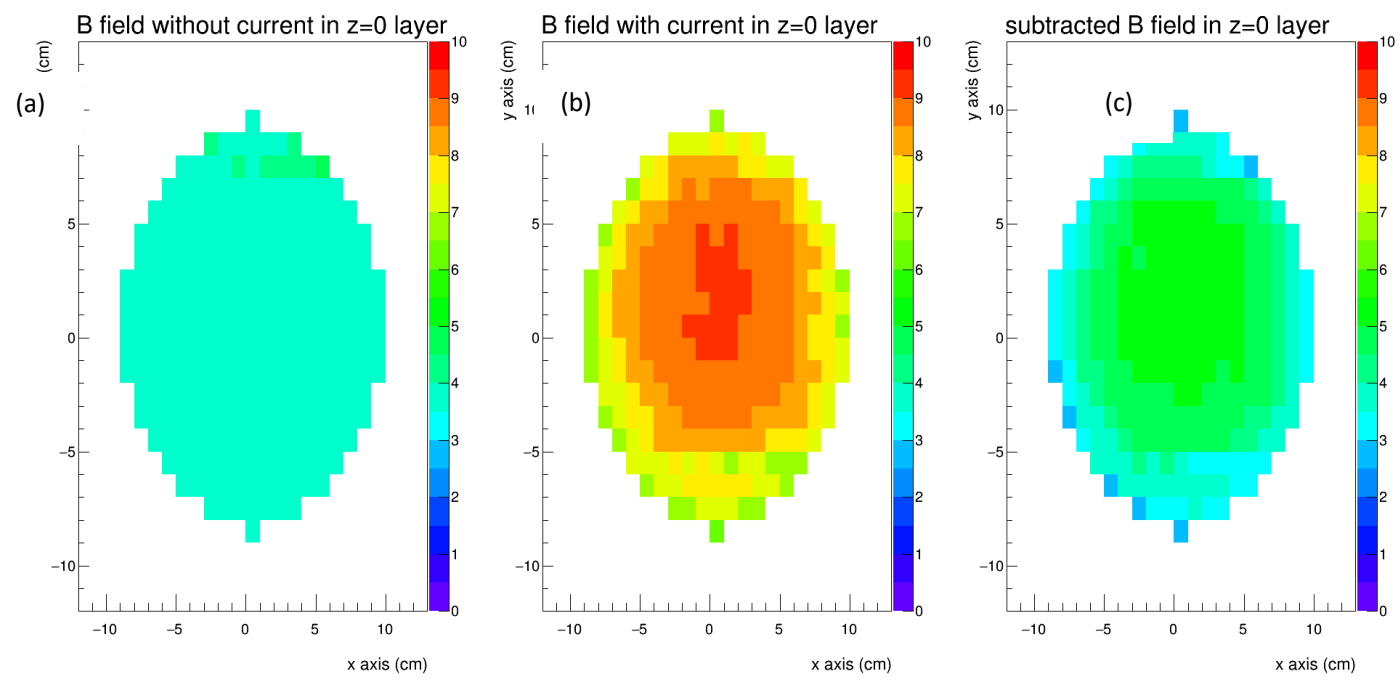

Figure 5. Measured Magnetic field data for the layer at $z=0(x, y, 0)$; figure left to right: magnetic field without applying current (a), the magnetic field with current (b), and difference of magnetic field with and without current (c).

As it is expected and shown in Fig. 5, there is a uniform magnetic field distribution in the center of the cylinder when there is no current as shown in Fig.5 (a) but when the current is applied, the maximum magnetic field is measured at the center of the cylinder as in Fig.5 (b) and it decreases towards the edges. However, as we subtract the background magnetic field data from the total magnetic field with current, a uniform transient magnetic field graph was obtained as shown in Fig. 5(c). In order to examine the change of the magnetic field in the cylinder according to the change in the $\mathrm{x}$ and $\mathrm{y}$ coordinates, the $\mathrm{z}$-axis is held constant at a certain point and the $\mathrm{x}$ and $\mathrm{y}$ axis are changed. The variation of the magnetic field at $\mathrm{z}=0(\mathrm{x}, \mathrm{y}, 0)$ coordinate with respect to changing $\mathrm{x}$ and $\mathrm{y}$-axis is given in Fig. 6 . There is no difference between the variation of the magnetic field with respect to $\mathrm{x}$ or $\mathrm{y}$-axis ( $\mathrm{z}$ is fixed) because one represents the horizontal and the other represents the vertical direction. As it is presented in Fig. 6(a) and Fig. 6(b) when the sensor moved from the inner tangent surface of the cylinder $(x=-9)$ from one side to the other inner tangent surface $(x=+9)$, magnetic field increases toward the center $(\mathrm{x}=0)$ first and becomes maximum at the center, then it starts to decrease to the inner side surface. Theoretically, the change of a magnetic field is expected to draw a curve along the $\mathrm{x}$ or $\mathrm{y}$-axis (-10 to 10). However, there is not a uniform line but deviations due to the millimetric deviations during the movement of the robot arm. The background magnetic field measurement data along the x-axis are as shown in Fig.6 (a). Since the electric current is not applied, it is in the form of a straight line from top to bottom both for $\mathrm{x}$ and $\mathrm{y}$ axes.
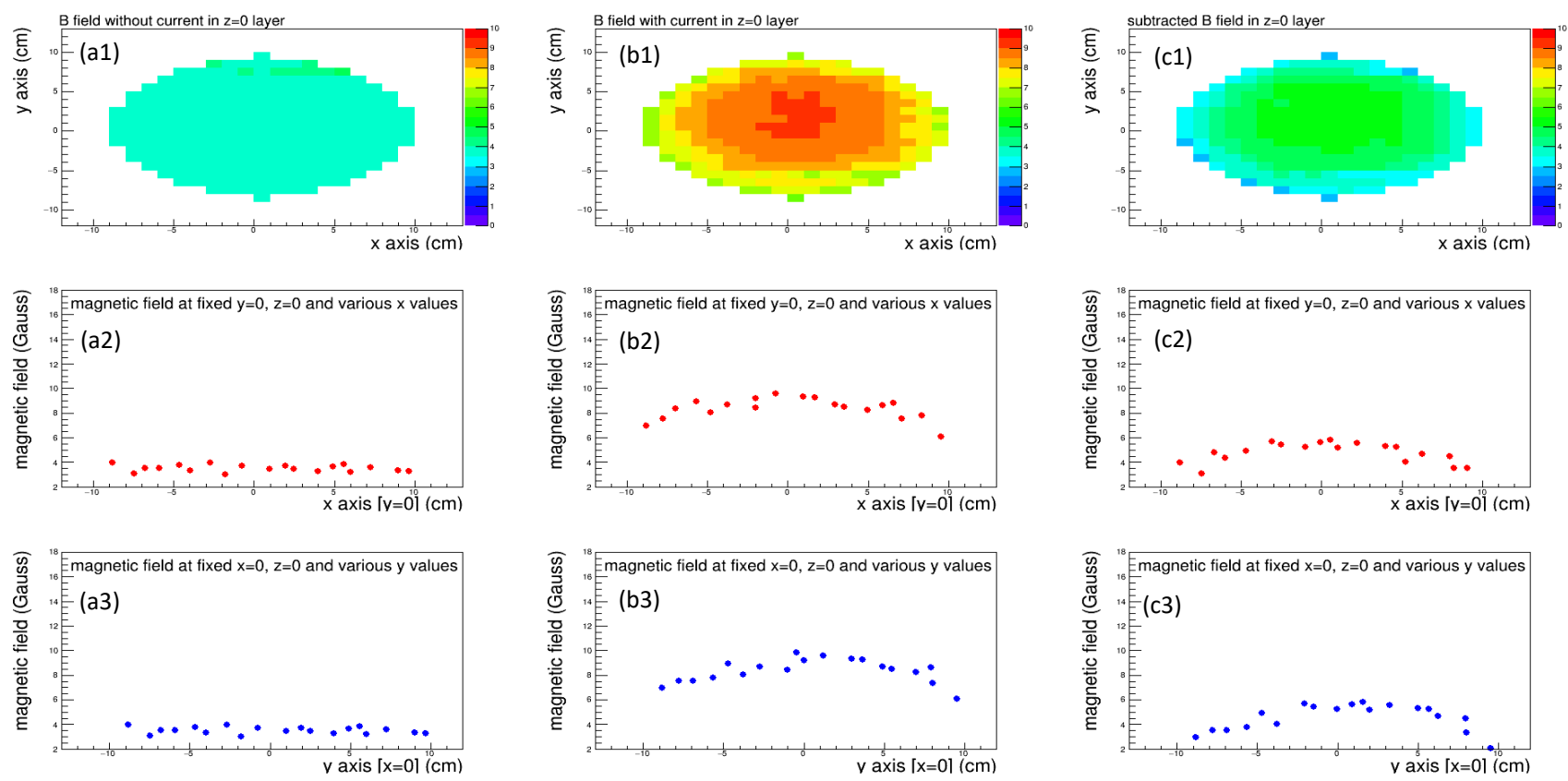

Figure 6. Magnetic field data for the layer at $\mathrm{z}=0(\mathrm{x}, \mathrm{y}, 0)$; Figure left to right: background magnetic field (a1-a3), magnetic field with current (b1-b3) magnetic field difference with and without current (c1-c3) 
The $\mathrm{x}$ and $\mathrm{y}$ coordinate axis is kept constant and the magnetic field distribution due to the change of the $\mathrm{z}$-axis is given in Fig. 7. The first Fig. 7(a) is the magnetic field data, measured without applying the current and it is around 4 Gauss. The second Fig. 7 (b) is the measured magnetic field data after the current is applied to the system. The magnetic field increases first along the $\mathrm{z}$-axis and reaches the highest value in the middle and becomes maximum at 14 Gauss and then starts to decrease again. Fig. 7(c) represents the real magnetic field from the application of current to the coil. The magnet was cooled by passing water through the pipes and water circulation was performed with the help of a pump but no difference was observed between the measurements as the pipes weren't overheated.

magnetic field at fixed $x=0, y=0$ and various $z$ values

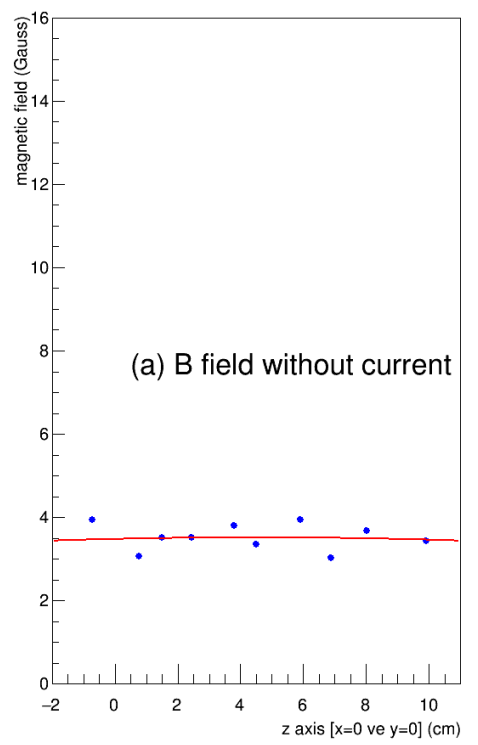

magnetic field at fixed $x=0, y=0$ and various $z$ values

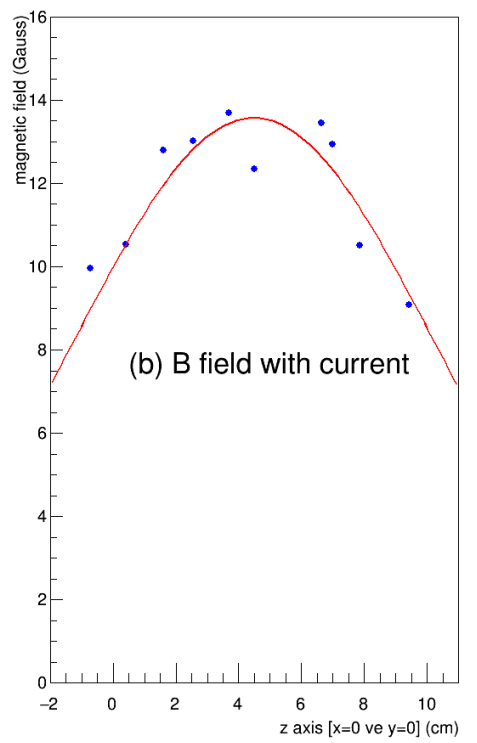

magnetic field at fixed $x=0, y=0$ and various $z$ values

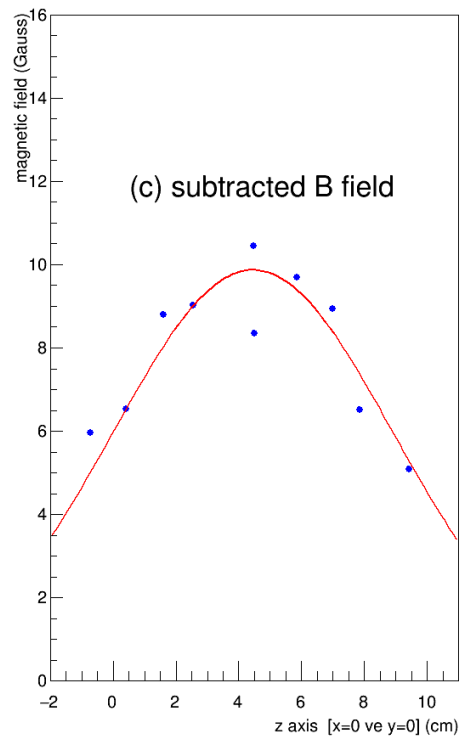

Figure 7. Magnetic field distribution with respect to the z-axis $(0,0, z)$; Figure left to right: magnetic field without applying current, magnetic field with current, and magnetic field difference with and without current.

The z-axis was divided into 10 equal parts with $1 \mathrm{~cm}$ intervals along the center of the cylinder, and the magnetic field measurement performed in each slice along the $\mathrm{x}$ and $\mathrm{y}$ axes. The magnetic field intensity increases from the outside to the center of the cylinder along the z-axis as shown in Fig. 7 (c). Magnetic field intensity data at $z=5(x, y, 5)$ with respect to $x$ and $y$-axis is presented in Fig.8. The magnetic field at the inner tangent surfaces of the cylinder is measured higher than the middle parts. The magnetic field on the zaxis appears to be lower than the magnetic field near the inner surfaces of the cylinder. Normally, the magnetic field must be reduced from the z-axis to the inner tangent surface, but the field strength increased about by 2 Gauss as shown in Figure 8 (b). The body of the cylinder is made of 1010 iron steel and it is a ferromagnetic material. When current is applied to the solenoid magnet, the current coil increases the magnetic field intensity in the iron yoke, and the magnetic field intensity becomes maximum in the iron yoke compared to along the z-axis. The solid portions of the cylinder act as a source of a strong magnetic field, and causes an increased magnetic field area near the tangent surface of the cylinder. As it is seen in Fig. 8 (b), the magnetic field decreases along the $\mathrm{x}$-axis from $\mathrm{x}=0$ to $\mathrm{x}=$ $10 \mathrm{~cm}$ then it starts to increase again towards the inner tangent surface of the cylinder. When it moves to the other side of the cylinder from $\mathrm{z}=5$ to $\mathrm{z}=9$, the ferromagnetic domain begins to disappear along the $\mathrm{z}$-axis and finally, magnetic field distribution becomes like in Fig.6. 

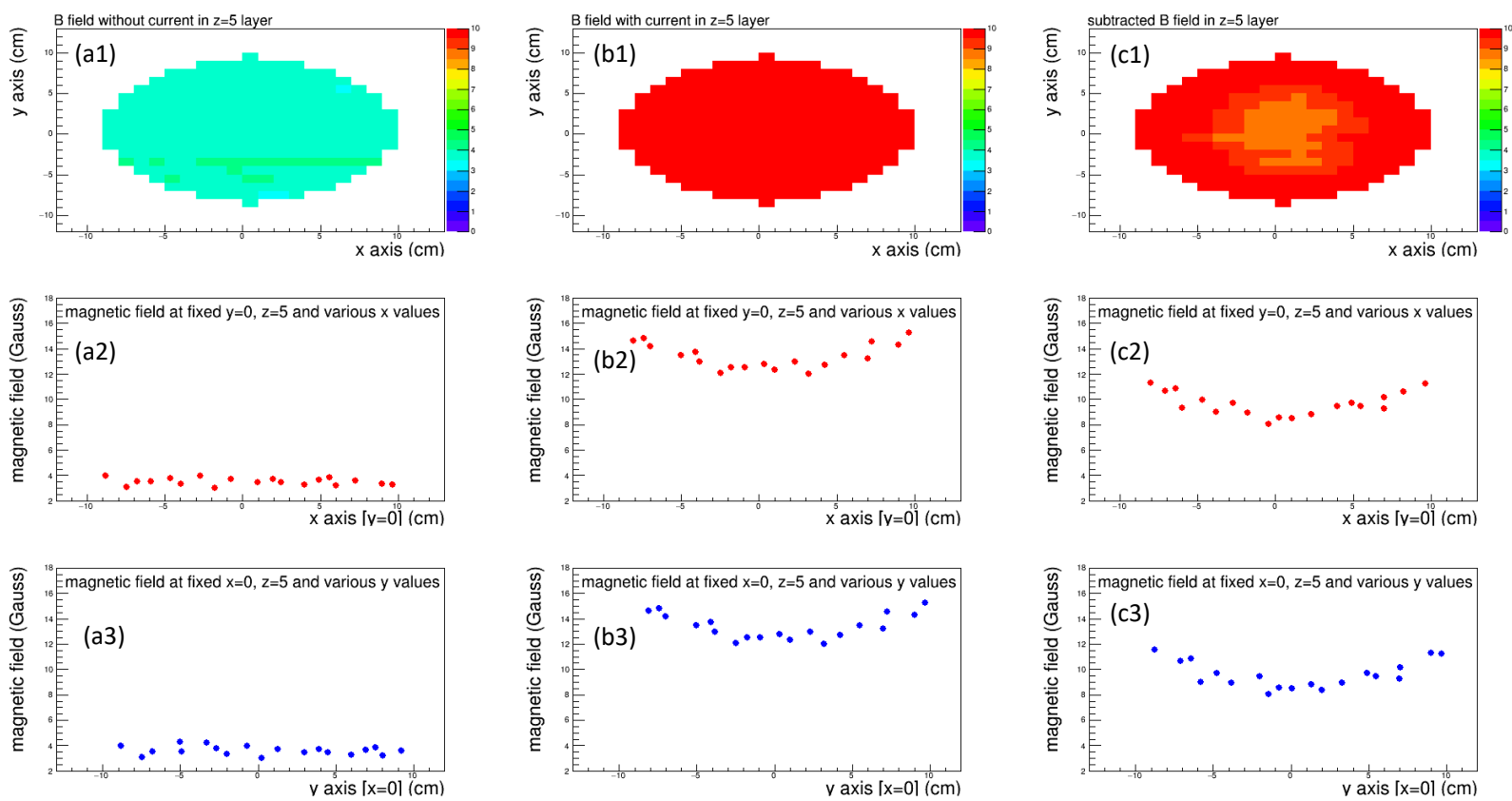

Figure 8. Magnetic field data for the layer at $z=5(x, y, 0)$; Figure left to right: background magnetic field (a1-a3), magnetic field with current (b1-b3) magnetic field difference with and without current (c1-c3)

\section{Numerical Approach}

A commercial version of The Flexible Generalized Minimal Residual method (FGMRES) solver (built-in COMSOL) was chosen because it is an iterative method for the numerical solution of a nonsymmetric system of linear equations. This method approximates the solution by the vector in a Krylov subspace with minimal residual (GMRES, 2019). FGMRES generates a converging solution by minimizing at each iterative cycle of the Euclidean norm of the system residual (Saad, 1993). The computation was performed on a server with 32 processors and 64 GB RAM. Computation was conducted for an unstructured mesh with a tetrahedron mesh type. Extremely fine mesh type was applied to the model and totally 244,301 tetrahedral, 90,322 triangular mesh element was obtained. Mesh quality was checked in terms of skewness which is based on the equiangular skew that penalizes elements with large or small angles as compared to the angles in an ideal element (Gothäll, 2019). Quality of 1 indicates the best possible element (Comsol, 2019), on the other hand, 0 represents a degenerated element (Sogukpinar, 2019; 2020a; 2020b). The average element quality for the current study was calculated as 0.66. Final mesh distribution for the solenoid magnet is given in Fig.9.

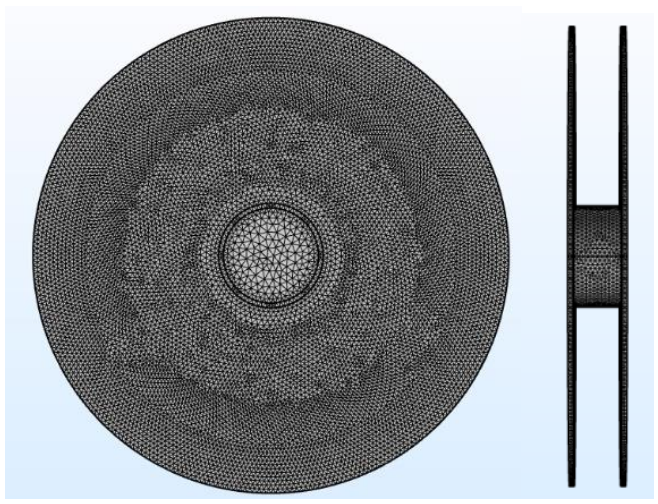

Figure 9. Mesh distribution for the water-cooled solenoid magnet

\section{Numerical results}

Magnetic flux density was calculated with commercial software COMSOL and front and side view central slice image is presented in Fig. 10. The magnetic field flux density is calculated as the highest in the center, and starts to decreases first and becomes minimum at a certain distance from the center and starts to increase up to the inner side tangential surface of the iron body. Because magnetic field flux density in the iron is much higher than the air and it is affecting the near-surface. 

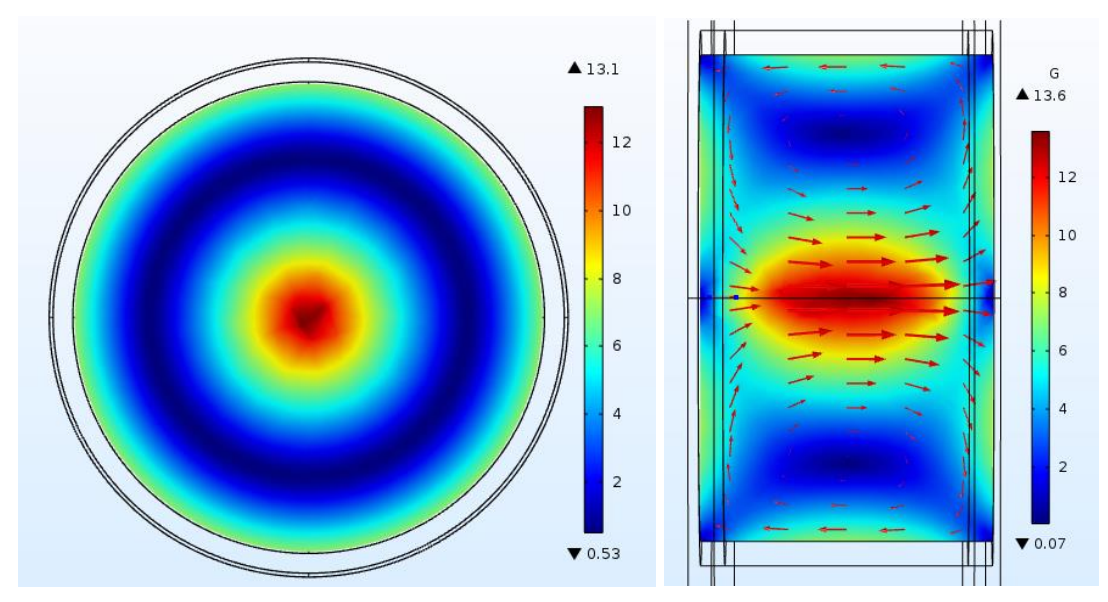

Figure 10. Magnetic field distribution on the central slice; Figure left to right: front view, and side view (Gauss)

Magnetic flux density was calculated and compared with the experimental measurement along the $\mathrm{z}$-axis and through the $\mathrm{X}$-axis at the central part of the solenoid magnet and is presented in Fig. 11. The magnetic field along the $\mathrm{z}$-axis has increased and reached its highest value in the center and started to decrease again. There is a good correlation between the experiment and the numerical calculation. However, according to the calculation result along the x-axis, there are some differences between the experiment and the numerical result close to the inner tangent surface of the cylinder. In the design of an ideal solenoid magnet with iron yoke, the magnetic field along the $\mathrm{x}$-axis must first decrease slightly and then increase again to the edge due to the ferromagnetic effect of the side surface as it is shown in Fig. 11(b). On the other hand, experimental magnetic field data showed a good correlation until distance at $\mathrm{x}=6 \mathrm{~cm}$ then deviation started until the inner surface of the magnet. The main reason for these differences is that the iron body is not smooth enough in the production process and experimental data reveal errors in the manufacturing process. Experimental data varies between 16 and 8 Gauss. However, numerical results vary between 1 and 17 Gauss.
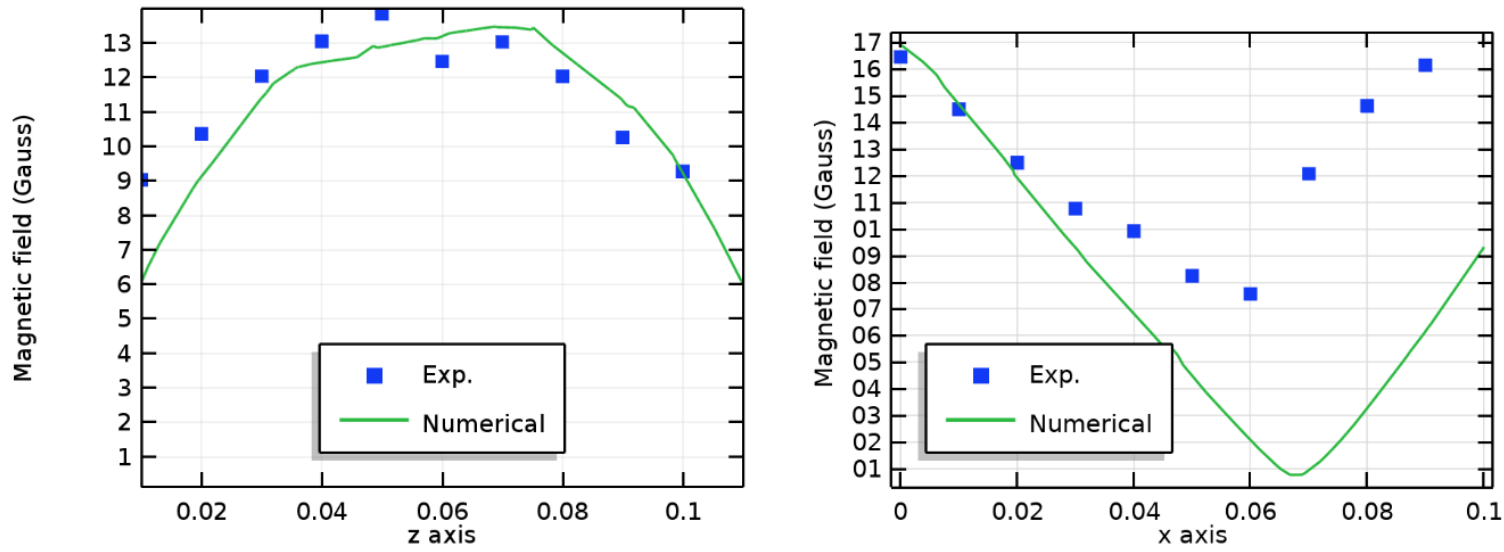

Figure 11. Magnetic flux norm density; figure left to right: along the $\mathrm{z}$-axis, along the $\mathrm{x}$-axis.

\section{Conclusion}

In this study, magnetic field mapping was performed for prototype water-cooled solenoid electromagnet, which was designed and produced for MDIS ion source in the Turkish Accelerator Center by using Hall sensor, which is attached to a robotic arm. Measurements were made in two stages. In the first stage, position and magnetic field values were measured without applying current to the solenoid magnet. In the second stage, position and magnetic field values were measured with applying current to the solenoid magnet. Magnetic field intensity was measured layer by layer perpendicular to the $\mathrm{z}$-axis. Measurements were started from the first layer at $\mathrm{z}=0$ and measurements were performed on a total of 10 layers with $1 \mathrm{~cm}$ intervals. In each layer, 257 measurements were performed along the $\mathrm{x}$ and $\mathrm{y}$-direction. The magnetic field measurement without applying electric current is approximately the same and the average of all the data was calculated as 3.72 Gauss. Magnetic field results after current applied to the solenoid magnet were analyzed and the average was calculated as 12.0 Gauss. Also, a numerical calculation was conducted for the same solenoid magnet and obtained data were compared with the experiment. For the numerical results, the same parameters with the experiment were used and the magnetic field 
was calculated in the core of the solenoid magnet and compared with the experiment. There is a good correlation between experiment and the numerical data along the $\mathrm{z}$-direction but deviations were observed in the $\mathrm{x}$ and $\mathrm{y}$-direction.

\section{Acknowledgment}

This work was supported by the Adiyaman University Scientific Research Project (Project no: FEFLTP/2017-0001).

\section{References}

Algin, E., Cetinkaya, H., Akkus, B., Sahin, L. (2014). TAC Proton Accelerator Facility: Normal Conducting Part. Proceedings of IPAC, Dresden, Germany.

Becker, R. (1990). Magnetic fields calculated by Intmag compared with analytical solutions and precision measurements. Nuclear Instruments and Methods in Physics Research Section A: Accelerators, Spectrometers, Detectors and Associated Equipment, 298(1$3), 13-21$.

COMSOL, How to Inspect Your Mesh in. (2019). Author Retrieved from https://www.comsol.com.

Feder, T. (2010). Accelerator school travels university circuit. Physics Today, 63 (2), 20-22.

Gothäll, H. (2019). How to Inspect Your Mesh in COMSOL Multiphysics. Author Retrieved from https://www.comsol.com.

GMRES, Generalized minimal residual method. (2019). Author Retrieved from https://en.wikipedia.org.

Jain, S. K., Naik, P. A., \& Hannurkar, P. R. (2010). Design, fabrication, and characterization of a solenoid system to generate magnetic field for an ECR proton source. Sadhana, 35(4), 461-468.

Lee, D. W., Hwang, K. P., \& Wang, S. X. (2008). Fabrication and analysis of high-performance integrated solenoid inductor with magnetic core. IEEE Transactions on Magnetics, 44(11), 4089-4095.

Maimone, F., Gammino, S. L., Celona, G., Ciavola, D. Mascali, N. Gambino, R. Miracoli, F. Chines, G. Gallo, S. Passarello. (2011). Commissioning of the New Versatile Ion Source (VIS) for High Power Proton Accelerators. PACS: 29.25._t 52.50.Sw 07.77.Ka.

Saad, Y. (1993). A flexible inner-outer preconditioned GMRES algorithm. SIAM Journal on Scientific Computing, 14 (2), $461-469$.

Sogukpinar, H. (2019). Seasonal temperature variation of solar pond under Mediterranean condition. Thermal Science, 23(6A), $3317-$ 3326.

Sogukpinar, H. (2020a). Numerical study for estimation of temperature distribution in solar pond in diverse climatic conditions for all cities of Turkey. Environmental Progress \& Sustainable Energy, 39(1), pp 1-12.

Sogukpinar, H. (2020b). Numerical Investigation of Influence of Diverse Winglet Configuration on Induced Drag. Iranian Journal of Science and Technology, Transactions of Mechanical Engineering, 44(1):203-215.

TAEK-Turkish Atomic Energy Agency. (2012). Proton Accelerator Facility. Author Retrieved from http://www.taek.gov.tr/.

TAC, Turkish Accelerator Center project. (2019). Author Retrieved from http://thm.ankara.edu.tr. 